\title{
A comparison between two types of central venous catheters in the prevention of catheter- related infections: the importance of performing all the relevant cultures.
}

\author{
J van Vliet ${ }^{1, *}$, JA Leusink¹, BM de Jongh ${ }^{2}$, A de Boer ${ }^{3}$
}

'Department of Anaesthesiology and Intensive Care, 'Department of Microbiology,

St. Antonius Hospital, Nieuwegein, The Netherlands. 3Department of Pharmacoepidemiology and Pharmacotherapy, Utrecht Institute of Pharmaceutical Sciences, University of Utrecht, The Netherlands ${ }^{3}$

*Author for correspondence: Leyenburg Hospital, Department of Anaesthesiology and Intensive Care, PO Box 40551, 2504 LN, The Hague, The Netherlands. Tel: 0703592000

Accepted 17 March 2001

Clinical Intensive Care 2001, 12(3): $135-140$

\begin{abstract}
Objective: to determine the efficacy of double-lumen central venous catheters coated with chlorhexidine and silver-sulfadiazine in reducing the incidence of catheter-related infections.

Design: a randomized controlled trial.

Setting: medical-surgical intensive care unit of a 600-bed teaching hospital.

Patients: adult patients who needed a central venous catheter in the subclavian vein. Intervention: patients received either a standard catheter $(n=46)$, or a chlorhexidine and silver-sulfadiazine-impregnated catheter $(n=48)$.

Measurements: catheters were removed if there was no need for them anymore or if they were suspected as a cause of infection. Three parts of the catheter were cultured semi-quantitatively. Blood-, wound-, urine- and sputum-cultures were obtained on the day of catheter removal in order to allow a correct diagnosis of catheter-related bloodstream infection (CR-BSI).

Results: in total 16 catheters were colonized (17\%), six in the antiseptic-bonded catheter group $(12 \%)$ and 10 in the standard catheter group ( $22 \%)$; relative risk $0.51(95 \% \mathrm{CI}$ $0.17-1.65), p=0.24$. After adjustment for potentially confounding factors, relative risk was $0.42(95 \% \mathrm{Cl} 0.12-1.49), p=0.18$. In five out of 16 colonized catheters, there were positive blood cultures with the same microorganism cultured from the catheter as well. In these five patients the diagnosis of CR-BSI was uncertain as in four patients there were other positive cultures with the same micro-organism (from wound, urine or sputum) on the day of catheter removal and in one patient the same micro-organism was already cultured from the blood the day prior to catheter insertion.

Conclusion: a protocol in which the catheter is inserted in the subclavian vein results in a low percentage of colonization. There was a trend towards less colonization in the chlorhexidine and silver-sulfadiazine-impregnated catheter group, but this was not statistically significant. In our study-population there were no proven cases of CR-BSI. It is essential to culture wounds, sputum and urine, as well as blood on the day of removal of the catheter to prevent over diagnosis of catheter-related bloodstream infections.
\end{abstract}

\section{Introduction}

Catheter-related infections form a serious complication of indwelling central venous catheters and are responsible for an important percentage of nosocomial infections in intensive care units. The incidence of catheter-related infections is estimated to be between $1 \%$ and $14 \%$ of all inserted catheters, ${ }^{1.23}$ with an associated mortality rate of about $10-25 \% .^{3,4,3.6}$
Moreover, these infections add significantly to the costs of treatment and length of hospital stay. ${ }^{6.7 .8}$ It is difficult however, to compare various studies because the definition of the different clinical conditions is not uniform. ${ }^{2}$ The Centres for Disease Control and Prevention have proposed definitions for catheterrelated infections. This should facilitate comparisons between various studies. 
Numerous factors influence the incidence of catheter-related infections, such as site of insertion, material of which the catheter is made, duration of insertion, single vs. multi-lumen catheters and type of dressing applied. ${ }^{2,3,4,7,10,11}$ A reduction in catheterrelated infections can possibly be accomplished using catheters coated or impregnated with antimicrobial agents. In the last couple of years, several types of these catheters have been developed and studied. ${ }^{1,6,12,13}$ Maki et al. found a significant reduction in bloodstream infections comparing a chlorhexidine and silversulfadiazine-coated catheter with a standard catheter.'

In a prospective randomized trial, we investigated the difference in catheter-related infections between this type of catheter, made of polyurethane (Arrowgard, Arrow International, Reading, PA, USA) and a standard catheter, made of the same material with a hydrophilic coating (Hydrocath, Ohmeda, Swindon, UK ). Catheters were inserted in the subclavian vein, into intensive care patients who had the need for a central venous catheter for more than 3 days.

\section{Material and methods}

Our randomized controlled trial was conducted at the intensive care unit (ICU) of the St. Antonius Hospital, a 34 bed medical-surgical unit, with an emphasis on major vascular and cardio-thoracic surgery. On admission to the ICU, the central venous catheter is placed in the internal jugular vein in the majority of patients. According to local protocol, this catheter has to be removed 3 days after insertion. If a central venous catheter is still necessary after this period, it is inserted in the subclavian vein. All adult patients who received a double-lumen central venous catheter in the subclavian vein after this period participated in the study. Approval of the institutional medical ethics committee was obtained. Since both products are commercially available and no deviation from routine clinical protocols was made, informed consent was waived. Using commercially available catheters, it was impossible for the investigators to be blind to the study. Patients were excluded if they were allergic to chlorhexidine or sulphur. A standard catheter was placed on even days, while an antiseptic-bonded catheter was placed on odd days.

Study catheters were inserted by house-officers, who wore masks, surgical gowns, sterile gloves and a cap. The insertion site was thoroughly cleaned with chlorhexidine-ethanol scrub, and large sterile drapes were used as sterile precautions. The catheter was then introduced percutaneously using the Seldinger technique and securely sutured. The protocol did not allow the changing of a catheter over a guide-wire. All sites were covered with an occlusive transparent dressing, which was changed on a regular basis.
For each patient, data collection included age, weight, height, sex, operative or non-operative admission, regular or emergency admission, use of antibiotics, reason for using the catheter, and duration of insertion of the catheter. On the day of insertion, the Acute Physiology and Chronic Health Evaluation II (APACHE II) score of all patients was measured.

The catheter remained in place until it was no longer needed, or until it was suspected of catheter-related infection. On the day of removal, cultures of blood, wounds, urine, and sputum were performed. The blood culture was always taken from a peripheral vein, since this is part of our hospital protocol. We also noted if a patient from our study-group died.

The entire catheter was removed aseptically, and the intravenous part of the catheter was cut into three pieces of approximately $3 \mathrm{~cm}$ each: a proximal segment closest to the skin, a middle segment, and the tip, which is the most distal segment of the catheter. These segments were cultured semi-quantitatively by using the roll-plate-method described by Maki. ${ }^{14}$ Using this technique, only the external surface of the catheter was cultured.

Catheter-related infection was divided in three different clinical entities. First: exit-site infection with swelling and erythema or purulence of the skin; Second: colonization of the catheter, when more than 15 colony forming units of any organism were cultured by the semi-quantitative technique; Third: catheter-related bloodstream infection (CR-BSI) when the same microorganism isolated from the catheter, was isolated from the blood as well, without other positive cultures."

\section{Statistical analysis}

Categorical variables were tested by the Chi square test (SPSS). Odds ratios with $95 \%$ confidence limits were estimated by logistic regression; these express the relative risk of colonization for the antiseptic-bonded catheter versus the standard catheter. First an univariate analysis was done (unadjusted relative risk), and subsequently potentially confounding factors (age, gender, APACHE-score, emergency admission, total parenteral nutrition, duration of catheterization, antibiotics on day of removal of the catheter) were entered into the model (adjusted relative risk).

\section{Results}

A total of 94 catheters were evaluated in this study: 48 in the antiseptic-bonded catheter group, and 46 in the standard catheter group. In Table 1, the characteristics of the patient population are shown. There were no significant differences between the two groups. In both groups, the percentage of post-operative patients was high, reflecting the nature of the $\mathrm{ICU}$. The mean 
Table 1. Characteristics of patients and catheters

\begin{tabular}{|c|c|c|c|}
\hline Variable & $\begin{array}{l}\text { Study catheter } \\
(n=48)\end{array}$ & $\begin{array}{l}\text { Control catheter } \\
(n=46)\end{array}$ & P value \\
\hline Age $(y \pm S D)$ & $67 \pm 8$ & $68 \pm 7$ & ns \\
\hline Male/ female $(n / n)$ & $29 / 19$ & $35 / 11$ & ns \\
\hline Post-operative $(\%)$ & 79 & 74 & ns \\
\hline Emergency admission (\%) & 61 & 67 & ns \\
\hline APACHE $2 \pm S D$ & $24 \pm 8$ & $22 \pm 7$ & ns \\
\hline Mortality $(\%)$ & 23 & 27 & ns \\
\hline $\begin{array}{l}\text { Mean duration of catheterization } \\
(d \pm \text { SD })\end{array}$ & $9 \pm 7$ & $10 \pm 7$ & ns \\
\hline Total parenteral nutrition (\%) & 31 & 35 & ns \\
\hline Antibiotics day removal (\%) & 46 & 48 & ns \\
\hline
\end{tabular}

APACHE II score and the percentage of patients who eventually died was high in both groups. The percentage of patients, who received antibiotics on the day of removal of the catheter, was the same in both groups. The percentage of patients who received total parenteral nutrition was not significantly different between the groups.

The mean duration of catheterization was 9.1 days in the antiseptic-bonded catheter group (range 1-39 days), vs. 10.2 days in the standard catheter group (range $2-28$ days). This difference was not statistically significant.

Of the 94 catheters in our study, a total of $16(17 \%)$ resulted in at least one of the segments colonized: six out of 48 catheters in the antiseptic-bonded catheter group (12\%), and 10 out of $46(22 \%)$ catheters in the standard catheter group ; relative risk $0.51(95 \% \mathrm{Cl} 0.17$ 1.65), $p=0.24$; relative risk when adjusted for confounding factors 0.42 (95\% CI $0.12-1.49), p=0.18$.
In Table 2, all microbiological results are shown for the colonized antiseptic-bonded catheters, as well as for the other cultures on the day of removal of the catheter. In Table 3, the same is done for the colonized standard catheters. These tables show that if only the tips had been cultured, in 12 out of 94 (13\%) catheters, colonization would have been diagnosed: four out of $48(8 \%)$ in the antiseptic-bonded catheter group, and 8 out of $46(17 \%)$ in the standard catheter group $(p=$ 0.31 ). When all the colonized segments between the two groups were compared, 13 segments in the antiseptic-bonded group were colonized and 23 segments in the standard catheter group were colonized $(p=0.18)$.

The microorganism most often isolated from the catheters was Coagulase Negative Staphylococci (CNS), in seven out of 16 catheters, as can be seen in Tables 2 and 3.

Table 2. All colonized chlorhexidine and silver-sulfadiazine-impregnated catheters and relevant other cultures on the day of removal of the catheter ${ }^{1}$

\begin{tabular}{|c|c|c|c|c|}
\hline $\begin{array}{l}\text { Study catheter } \\
\text { Arrowgard }\end{array}$ & Proximal part ${ }^{2}$ & Middle part & Tip & $\begin{array}{l}\text { Relevant } \\
\text { cultures } \\
\text { on day removal }\end{array}$ \\
\hline 1 & $\mathrm{CNS}^{3}$ & no cultures & no cultures & no cultures \\
\hline 2 & CNS & CNS & CNS & no cultures \\
\hline 3 & Candida sp. & Candida sp. & Candida sp. & $\begin{array}{l}\text { Various, } \\
\text { no Candida sp. }\end{array}$ \\
\hline 4 & Candida sp. & Candida sp. & Candidasp. & $\begin{array}{l}\text { Candida sp. in urine and } \\
\text { sputum cultures }\end{array}$ \\
\hline$\sqrt{15}+x^{2}+x^{2}$ & E. faecalis & E. faecalis & E. faecalis & $\begin{array}{l}\text { E. faecalis in blood } \\
\text { and wound cultures }\end{array}$ \\
\hline 6 & no cultures & no cultures & $\begin{array}{l}\text { K. } \\
\text { pneumoniae }\end{array}$ & $\begin{array}{l}\text { K. pneumoniae } \\
\text { in blood, urine, } \\
\text { and sputum cultures }\end{array}$ \\
\hline
\end{tabular}

1 The catheters are not represented in chronological order. ${ }^{2}$ The segment of the catheter closest to the skin. ${ }^{3}$ Coagulase negative staphylococci. 
Table 3. All colonized standard catheters and relevant other cultures on the day of removal of the catheter ${ }^{1}$

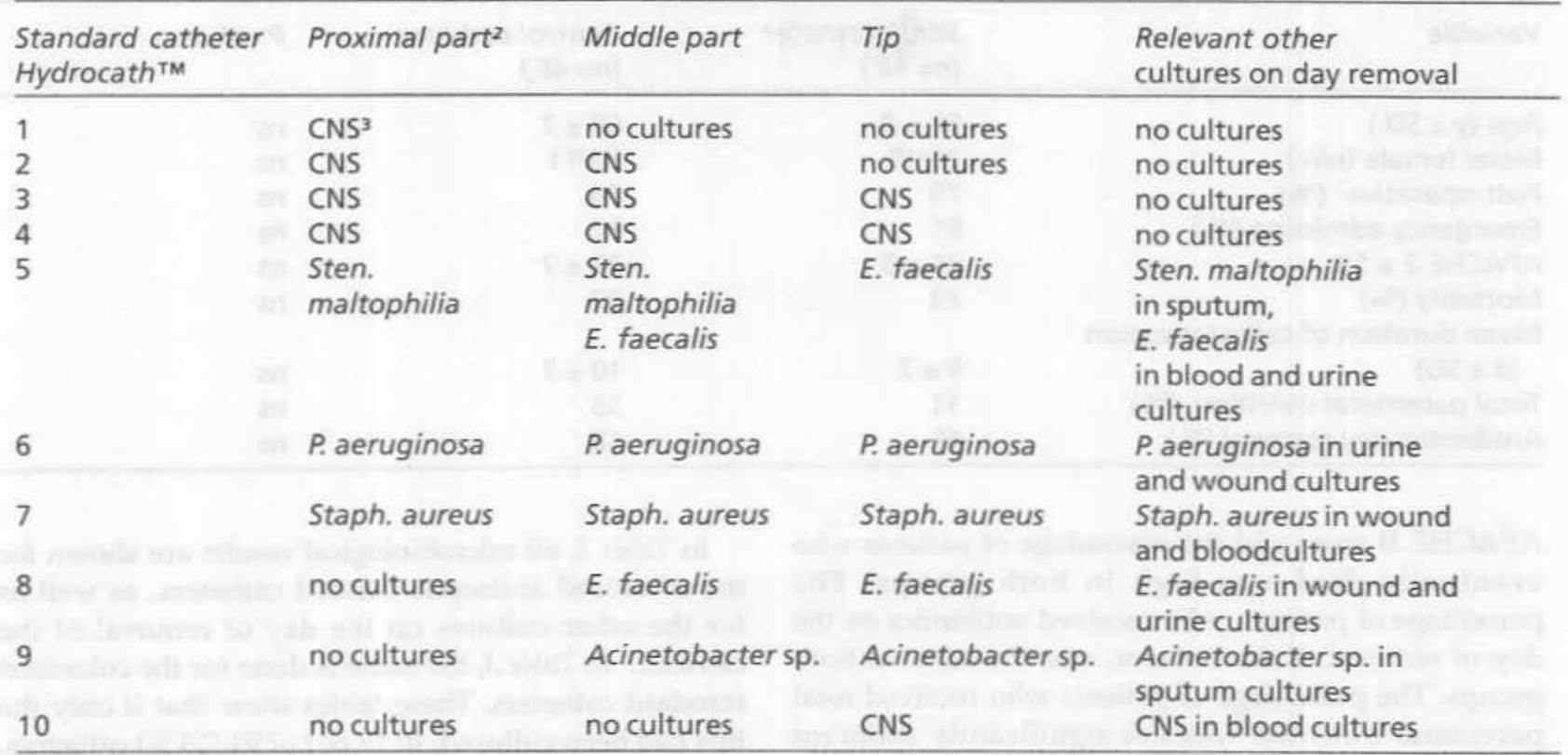

${ }^{1}$ Catheters are not represented in chronological order. ${ }^{2}$ The segment of the catheter closest to the skin. ${ }^{3}$ Coagulase negative staphylococci

In six out of seven cases, there were no positive blood or other cultures with CNS and in one case (standard catheter number 10), CNS were already isolated from a peripheral blood culture 1 day before placement of the catheter. Because of fever and suspicion of CR-BSI, this catheter was removed 2 days after placement. According to the definition, there was no CR-BSI in this case, since the catheter became colonized due to a pre-existing bacteraemia.

In nine cases, other microorganisms than CNS were responsible for the colonization of the catheter (Tables 2 and 3). In eight out of nine cases, this same microorganism could be isolated from other cultures, taken on the day of removal. In one case (antisepticbonded catheter number 3), Candida-species were isolated from the proximal and middle segment of the catheter, with no other cultures of this microorganism on the day of removal.

In five out of 16 catheters, the same micro-organism was isolated from the blood as well as from the catheter, but in four out of five catheters, the same microorganism could be isolated from other cultures as well (study catheter number 5 and 6, standard catheter number 5 and 7 ). The fifth case was the previously mentioned standard catheter number 10 . This means that in our study-group the possibility remains that the bacteraemias were caused by infections other than the colonized catheters, implicating that there were no proven cases of CR-BSI.

We found two positive blood cultures on the day of removal of the catheter, both of them in the antiseptic- bonded catheter group, without colonization of the catheter or other positive cultures with the same microorganism.

\section{Discussion}

Our protocol, which mandates insertion of catheters in the subclavian vein after 3 days resulted in a relatively low percentage of catheter colonization, especially when only the positive tips were taken into account $(13 \%)$, compared to other studies. ${ }^{16}$ We cultured three parts of the catheter in an attempt to establish the pathway of colonization, but no firm conclusions could be drawn from these results. A disadvantage of routinely inserting catheters in the subclavian vein might be the development of complications other than infections, for instance pneumothorax or haematoma, outweighing the benefits of colonization-reduction.

We could not demonstrate any proven CR-BSI in our study, implicating that there was no significant difference between chlorhexidine and silversulfadiazine-impregnated catheters and hydrophobic standard polyurethane catheters in the incidence of serious catheter-related infections in our population. Although the total number of colonized segments in the standard catheter group was higher than in the antiseptic-bonded catheter group, this was not statistically significant. These results partly conform with the results of Heard et al., who could find a significant difference in colonization of the catheters, but could not find a significant difference between the 
chlorhexidine and silver-sulfadiazine-coated catheter and a standard catheter in catheter-related bacteraemia. ${ }^{11}$ In their study-group, the incidence of colonization was very high, which was explained by culturing both tips and intradermal segments, and submaximal sterile precautions. In our view, an alternative explanation might be the fact that only slightly more than $50 \%$ of the catheters were placed in the subclavian vein. This vein is considered safest in preventing catheter-related infections.,10.11 Another factor was the use of double-lumen catheters in our study, compared to triple-lumen-catheters in theirs. The amount of lumina is another well-known risk factor for catheter-related infection. ${ }^{2.4}$ The third difference is changing a catheter over a guide-wire, which was considered safe by the authors. However, a metaanalysis by Cook and Randolph showed that there was a trend towards a higher rate of infection when changing catheters over guide-wires compared to new placement of the catheter. ${ }^{15,17}$ Interestingly, in the study by Maki et al., in eight out of 11 patients with a catheterrelated bloodstream infection, the catheter had been changed over a guide-wire. ${ }^{1}$ This is the only study that we know of in which a significant reduction in both the colonization of the catheter as in the amount of bloodstream infections was found, comparing the chlorhexidine and silver-sulfadiazine-coated catheter with a standard catheter. In their study population, the catheter was inserted in the subclavian vein in only $60 \%$ of the patients, using triple-lumen catheters. The results of the studies of Tennenberg et al. ${ }^{18}$ and Hannan et al. ${ }^{19}$ were comparable to the results of the study of Heard: a significant reduction in the colonization of the catheter, but no significant reduction in catheterrelated sepsis. In the study of Tennenberg et al. however, the population did not exclusively consist of intensive care patients and no mention was made of the cathetersite, an important risk-factor, as stated above. Moreover, the mean duration of catheterization was only slightly more than 5 days, which was relatively short. The incidence of colonization in the study of Hannan et al. was quite high, which was probably related to the very high percentage of catheters in central veins other than the subclavian (83\%). Two other studies, comparing antiseptic-bonded catheters with standard catheters, could not find significant differences in catheter-related infections between the two groups. It must be stressed however, that these catheters were solely used for total parenteral nutrition in patients who were not admitted to the ICU. ${ }^{5,20}$ The conclusions from a recent metaanalysis by Veenstra et al. in which all of the above studies were mentioned, were that central venous catheters impregnated with chlorhexidine and silversulfadiazine appear to be effective in reducing the incidence of both colonization of catheter and bloodstream infections in patients at high risk. ${ }^{16}$
The power of the present study is small because of the relatively small size of the study, but this is the case with the majority of the other studies. In the metaanalysis by Veenstra, it is concluded that a trial with adequate power would require more than 2000 catheters in each group. ${ }^{16}$ It is almost impossible to obtain such a large number of catheters in a single hospital.

In our study, as in the other studies mentioned, the catheters were cultured using the semi-quantitative roll-plate technique, ${ }^{14}$ in which only the external surface was cultured. This means that infections via connection hubs and infusion fluids could not be detected, since in these pathways of infection, the microorganisms migrate along the intraluminal surface of the catheter. Hub contamination is more common in catheters which are in place for more than 10 days, ${ }^{21}$ while contamination of the infusion fluids is a rare cause for catheter-related infection. ${ }^{22}$ It might have been possible that the two positive blood cultures on the day of removal of the catheter, and for which no explanation was found, were caused by intraluminal migration of microorganisms. The duration of catheterization in these two cases was about 7 days. If we had cultured the intraluminal surfaces of the catheters as well, as described by Sheretz and Raad et al. ${ }^{12,23,24}$ we might have found a greater percentage of colonization, and possibly catheter-related bacteraemia or sepsis. Our results, and culturing catheters only using the roll-plate-method in general, are probably an underestimation of the real incidence of catheterrelated infection. But, as Heard et al. already pointed out, "it is unlikely that culturing the internal surface of the catheter as well would have made much difference in the comparison of the two types of catheter, since the coating is only on the external surface of the catheter, and the rate of luminal colonization is expected to be the same in both groups.

If only blood cultures had been done on the day of removal of the catheter, the percentage of catheterrelated bacteraemia or sepsis in our study group would have been $4.3 \%$, since in four out of 16 catheters, the same micro-organism was cultured from the blood as well as from the catheters. This is comparable to the results of other studies. ${ }^{16}$ In these four cases, however, other relevant cultures on the day of removal of the catheter contained the same microorganism, making the diagnosis of CR-BSI uncertain. Systemic culturing might be a major difference between our study and various other studies, $1.5,12.16-19$ from which it does not become clear whether such cultures had been taken. This might also mean that a number of catheter-related infections in these studies were false positive. Our point of view is shared by Veenstra et al. who stated that the number of CR-BSI could have been overestimated in 
the various studies. ${ }^{16}$ Therefore, in order to make a correct diagnosis of catheter-related bacteraemia or sepsis, it is important to obtain other relevant cultures as well to prevent over-diagnosis of CR-BSI.

A recent comparison between chlorhexidine and silver-sulfadiazine-coated catheter and a rifampicinminocycline-coated catheter showed that the latter resulted in significantly lower rates of cathetercolonization and catheter-related-infection. These results suggest that we have not used the most effective antimicrobial-impregnated catheter. ${ }^{25}$

In conclusion, we have shown that a protocol in which double-lumen central venous catheters are inserted in the subclavian vein, results in a low percentage of colonization. In our study no significant difference was found between a chlorhexidine and silver-sulfadiazine coated catheter and a standard catheter in the incidence of catheter-related infections. There were no cases of proven CR-BSI. The main conclusion from this study is that sampling of only blood cultures on the day of removal of the catheter, could lead to a falsely high percentage of CR-BSI. This infection can only be diagnosed with certainty if cultures from other sites are negative.

\section{References}

1. Maki DG, Stolz SM, Wheeler S, Mermel LA. Prevention of central venous catheter-related bloodstream infection by use of an antiseptic-impregnated catheter. Ann. Intern. Med. 1997; 127: 257-266.

2. Raad I, Bodey GP. Infectious complications of indwelling vascular Catheters. Clin. Infect. Dis. 1992; 15:197-210.

3. Corona ML, Peters SG, Narr BJ, Thompson RL. Infections related to central venous catheters. Mayo Clin. Proc. 1990; 65: 979-986.

4. Reed CR, Sessler CN, Glauser FL, Phelan BA. Central venous catheter infections: concepts and controversies. Intensive Care Med. 1995; 21: 177-183.

5. Pemberton LB, Ross V, Cuddy P, Kremer H, Fessler T, McGurk E. No difference in catheter sepsis between standard and antiseptic central venous catheters. Arch. Surg. 1996; 131: 986-989.

6. Pittet D, Tarara D, Wenzel RP. Nosocomial bloodstream infection in critically ill patients:excess length of stay, extra costs, and attributable mortality. IAMA 1994; 271: 15981601

7. Raad I. Intravascular-catheter-related infections. Lancet 1998; 351: 893-898.

8. Arnow PM, Quimosing EM, Beach M. Consequences of intravascular catheter sepsis. Clin. Infect. Dis. 1993; 16: 778-784.
9. Pearson ML. Guideline for prevention of intravascular device-related infections. Am. J. Infect. Control 1996; 24: 262-293.

10. Mermel LA. Prevention of intravascular catheter-related infections. Ann. Intern. Med. 2000; 132: 391-402.

11. Heard SO, Wagle M, Vijakumar E, et al. Influence of triplelumen central venous catheters coated with chlorhexidine and silver-sulfadiazine on the incidence of catheter-related bacteremia. Arch. Intern. Med. 1998; 158: 81-87

12. Raad I, Darouiche R, Dupuis J, ef al. Central venous catheters coated with minocycline and rifampicin for the prevention of catheter-related colonization and bloodstream infections. Ann. Intern. Med. 1997; 127: 267-274.

13. Elliott $T$. Intravascular catheter-related sepsis- novel methods of prevention. Intensive Care Med. 2000; 26: S45S50.

14. Maki DG, Weise CE, Sarafin HW. A semiquantitative culture method for identifying intravenous-catheter-related infection. N. Engl. J. Med. 1977; 296: 1305-1309.

15. Randolph AG. An evidence-based approach to central venous catheter management to prevent catheter-related infection in critically ill patients. Critical Care Clinics 1998; 14: $411-421$.

16. Veenstra DL, Saint S, Saha S, Lumley T, Sullivan SD. Efficacy of antiseptic-impregnated central venous catheters in preventing catheter-related bloodstream infection. IAMA 1999; 281: 261-267

17. Cook D, Randolph A, Kernerman P, et al. Central venous catheter replacement strategies: A systematic review of the literature. Crit. Care Med. 1997; 25: 1417-1424.

18. Tennenberg S, Lieser M, McCurdy B, et al. A prospective randomized trial of antibiotic and antiseptic-coated central venous catheter in the prevention of catheter-related infections. Arch. Surg. 1997; 132: 1348-1351.

19. Hannan M, Juste RN, Umasanker S, et al. Antiseptic-bonded central venous catheters and bacterial colonisation. Anaesthesia 1999; 54: 868-872.

20. Ciresi DL, Albrecht RM, Volkers PA, Scholten DJ. Failure of antiseptic bonding to prevent central venous catheterrelated infection and sepsis. Am. Surg. 1996; 62: 641-646.

21. Linares J, Sitges-Serra A, Garau J, Perez JL, Martin R. Pathogenesis of catheter sepsis : a prospective study with quantitative and semiquantitative cultures of catheter hub and segments. J. Clin. Microbiol. 1985; 21: 357-360.

22. Maki DG. Infection Caused by Intravascular Devices: Pathogenesis, Strategies for Prevention. Royal Society of Medicine Services Ltd: London 1991.

23. Sheretz RJ, Raad I, Balani A, et al. Three year experience with sonicated vascular catheter cultures in a clinical microbiology laboratory. J. Clin. Microbiol. 1990; 28: 76-82.

24. Raad I, Sabbagh MF, Rand KH, Sheretz RJ. Quantitative tip culture methods and the diagnosis of central venous catheter-related infections. Diag. Microbiol. Infect. Dis. 1991; 15: $13-20$.

25. Darouiche RO, Raad II, Heard SO, et al. A comparison of two antimicrobial-impregnated central venous catheters. N. Engl. J. Med. 1999; 340: 1-8. 